\title{
Studying the Concept of Quantum Computing and Analysis of Its Components, Benefits and Challenges
}

\author{
Halkar Rachappa
}

Asst. Prof. \& Head Dept. of Computer Science

Govt. Commerce \& Management College,

BALLARI(Karnataka).

email :halkarrg@gmail.com

Abstract: - This paper will discuss about the use of quantum computing in the computer engineering field to make computers reliable and fast to perform various typical computations. It is the process of using concept of quantum theory in computing algorithms and other computations and calculations along with the use of computer systems. The computer system always understands the binary language of bits and bytes or in other words we can say that everything which is computed using computers should be first converted into stream of 0 's and 1's so that computer can understand and then perform the calculations. This will restrict the scope of certain calculations for the computers. Scientists and engineers have come together to implement the quantum computing along with computers and due to this they are able to make calculations which were not possible before its introduction. The quantum theory uses bits and qubits of quantum theory and allows them to be available in more than one state and make possible various typical calculations easy and fast. The paper will explain various types of quantum computing techniques and how they are useful for the organisations. The benefits and challenges of the quantum computing in the field of computer systems will also be discussed.

Keywords: - Quantum theory, Quantum computing and its types, Characteristics of Quantum Computing, Advantages and challenges, Components of quantum computing.

\section{Introduction: - [1]}

Quantum computing is the new technology which improves simple computer systems in to high tech systems which have the capability to perform various mathematical computations, physics computations, information theories etc. With traditional computer systems it is observed that the capability of systems to perform tough algorithms is very less and hence researchers started search to improve the calculation system of the computer system so that they can be used to perform other major difficult calculations which in turn will help the scientists to save their time and energy which they spend on performing manual scientific calculations. Old traditional computer systems understand the language of binary system which means they will first convert the human language into stream of bits and bytes and then perform the calculations and then give results in the language which is understood by the human beings. This makes limited calculations to be performed by the system. Where as on the other hand, Quantum computing helps to enhance the traditional computer system in to advance technology as it 
provides the capability to perform high computational power, fast speed which is calculated in terms of exponent, uses less power etc. The quantum computing uses the bits and qubits of quantum theory which makes it possible to perform most difficult calculations. In small traditional PCs, circuits with semiconductors, rationale entryways, and Integrated Circuits are unrealistic. Consequently, quantum computing utilizes the subatomic particles like molecules, electrons, photons, and particles as their pieces alongside their data of twists and states. They can be superposed and can give more mixes. Along these lines, they can run in equal utilizing memory effectively and thus is all the more impressive.

Importance of Quantum Computing: - [2]

$>$ The main importance of using quantum computing is that they are useful to reduce the power consumption to a great extent when compared to the traditional computer system.

They are much faster than the traditional computer systems as they use features of electron, atoms, photons etc and uses their bits and qubits.

$>$ They are used in the field of Artificial intelligence and helps to make machine learning process easy.

They are useful to perform complicated calculations faster and easily.

> Traditional computers can almost perform all types of calculations but they fail to perform complex scientific or mathematical calculations and hence quantum computing is been introduced to overcome this drawback of the traditional computer systems.

Properties or Characteristics of Quantum Computing: -

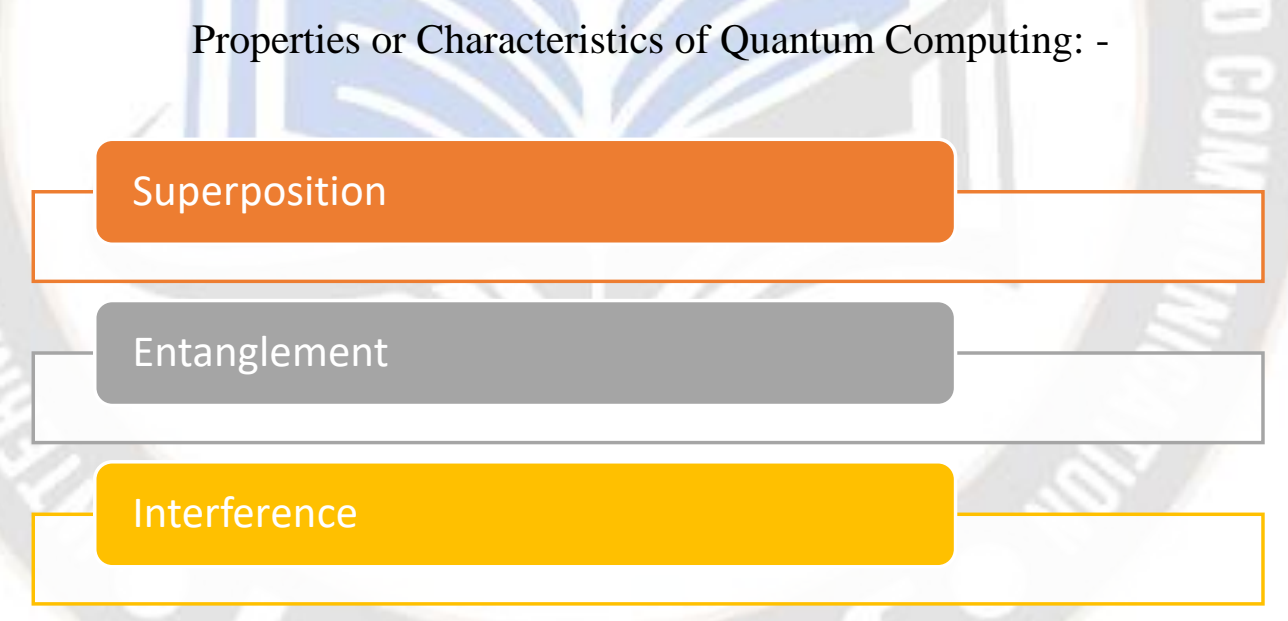

Figure 1 Properties of Quantum Computing.

The quantum computing uses three main properties of the quantum mechanics in order to store, perform calculations and operations on the data available. They are described as following: -

\section{Superposition: -}

* This is the most important property of the quantum mechanics which says that any quantum state present in the real environment is the combination of two or more than two quantum states.

* This describes the capability of the quantum system to represent that the qubit of quantum can exist in two different states at the same time.

*This helps in calculations which can be performed parallel to each other at very high speed without interfering with calculations of each other. 


\section{Entanglement: -}

* This is the most important property of the quantum computing as it will explain the physical properties of the systems.

*This helps to explain the relationship or dependency of two quantum qubits with each other.

*Once the qubits of quantum are entangled then it does not matter that how far they are present in the environment, the change in state of one qubit will definitely

effect the other qubit which is entangled with each other.

*This property helps to increase the computational power exponentially and saves a lot of time and efforts.

\section{Interference: -}

*Impedance is what is going on where mediation from clamour in the climate harms the quantum object, and furthermore the likelihood that the wave elements of particles can either build up or lessen one another.

*The quantum molecule might encounter obstruction. Assuming that every molecule goes through both the cuts (Young's twofold cut test) at the same time because of superposition, they can cross its own way disrupting the way bearing.

*The possibility of obstruction permits us to purposefully predisposition the substance of the qubit towards the required state. Be that as it may, it can likewise bring about a quantum PC to join its different calculations into one making it more blunder inclined.

Types of Quantum Computing: - [3]

Following are the three main types of Quantum Computers: -

\section{Quantum Annealer}

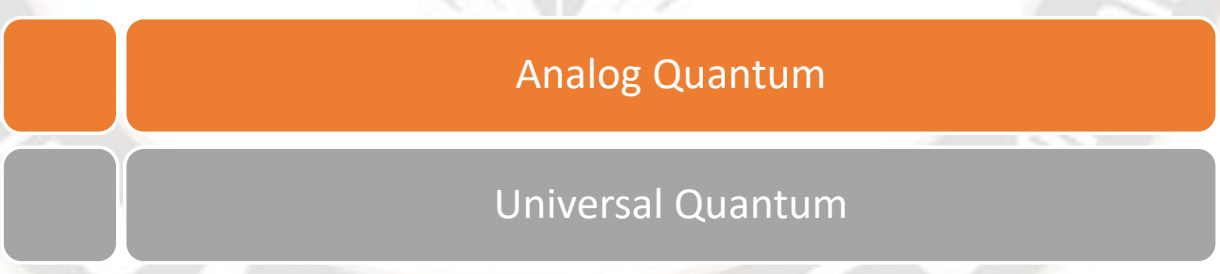

Figure 1 Types of Quantum Computing

1. Quantum Annealer: -

$>$ This type of quantum is less powerful than other quantum and has many drawbacks due to its restrictive nature.

This is the simplest form of quantum computer as it is easy to design it and has the capability to perform only one specific function.
$>$ It is similar to traditional computer systems due to which it is not very efficient to use.

$>$ It is used in optimization applications where global minimum of a function is identified over a number of solutions provided. 


\section{Analog Quantum: -}

$>$ It is the most powerful form of quantum computing which has the highest speed as compared to other quantum methods.

$>$ This type pf quantum computer will be able to perform complex problems and calculations which are not possible by using traditional methods of computing using computer systems.

$>$ The number of quits present in an analog quantum is somewhere near 50-100 as a result it gives fast results of computations.

$>$ Simple PCs the (numerical) issue to be addressed by building an actual framework that complies with similar limitations/regulations as the numerical issue. The responses are acquired by noticing and estimating the conduct of the actual reproduction.

$>$ The application where this technique is used are Sampling, optimization issues, Material Science etc.

\section{Universal Quantum: -}

$>$ This is one of the most powerful types of quantum computer.

$>$ It has the most complex technical implementation procedure and requires high technical skills in order to design it efficiently.

$>$ The qubits are more than 1000,000 due to which it has the highest computing speed of all the types of quantums available.

$>$ It is used in machine learning, Cryptography, Quantum chemistry etc where complex to typical calculations is required to perform.

$>$ The speed is calculated in terms of exponential form.

Thus, above are the main three types of quantum computers which are used to perform complex and difficult mathematical calculations. They help the scientists to perform various difficult calculations which will usually takes a lot of time to perform manually. In order to implement the concept of quantum computing with the traditional computers, it is essential that the designers should understand the requirements and have sound technical skills to implement the technique. The speed of the calculations and computations using quantum is based on the number of qubits the computer has. The higher the number of qubits in quantum computer, the higher the exponential speed of calculation done by it. It uses the concept of quantum mechanics in order to combine its features with the traditional methods. The difference between the traditional computer and quantum computer is that a quantum computer can perform all the tasks of the traditional computer but there are few complex calculations which cannot be performed by traditional computers but only by quantum computers.

Hardware and Software Need of the Quantum Computers: -

$>$ There is always requirement of the interface that act as an intermediate between the quantum and simple computer system which can then perform all the tasks.

The hardware requirement of the quantum computer is completed in four stages: -

a. Data plane: - In this space the qubit of the quantum computer is present.

b. Operations Plane: - This is responsible for performing all types of operations on the qubits of the quantum computers.

c. Control Processor plane: - This is responsible to identify the operations which need to be completed by another successive quantum.

d. Host Processor: - It is controlled by the traditional computer system which is responsible for controlling user 
interfaces, data structures, network requirements etc.

$>$ Like hardware requirements, the quantum systems also need software to run efficiently.

$>$ This will include having programming language that is used to perform testing and debugging of the system.

$>$ The languages used should be such that they are easily modified as and when required.

Components of Quantum Computers: - [4]

Following are the components of Quantum Computers: -

1. Qubits: - These are the memory units of the quantum computers. With the help of superposition property of quantum computers, it can hold numbers in the form of bits and qubits at the same time.

Qubits can be simulated by using one of the following approaches: -

$>$ Trapped icons: - The qubits which depends upon trapped icons is based upon optical signals which are transmitted through waves and delivered to the destined qubits.

$>$ Photons: - The main advantage of photon quantum is that it can be combined with fiber optic-based architecture. They can easily carry information in the form of qubits and can operate at room temperature.

$>$ Semiconducting material: - Qubits uses semiconducting materials like selenium, etc and the three main properties can be applied by using magnetic fields to it.

$>$ Superconducting material:

Superconducting qubit frameworks are controlled utilizing microwave and lowrecurrence electrical transmissions, the two of which are imparted through wires that run into cooling fridges to come to the qubits inside the controlled climate.
2. Quantum Registers: -

These registers will have the set of qubits and has the capacity to hold all possible combination of data provided at the same time.

3. Quantum Reversible Gates: -

$>$ The characteristics of reversible gates is that the input given can be identified by observing the out put of the system.

It is important that the quantum gates are reversible as quantum mechanics is reversible.

4. Quantum Processing unit: -

$>$ It is like CPU of the quantum computers which uses quantum rules to perform computations.

The parts of quantum processing units are:

\section{Quantum RAM}

Quantum Control Unit which operates the system.

Controller interface helps the communication between the CPU and QPU Advantages of Quantum Computing: - [5]

High exponential speed of calculation: With the integration of quantum mechanics in conventional computers the speed of computing complex calculations increases at a great rate.

$>$ The concept of quantum computing helps to understand and learn Artificial intelligence and machine learning easily.

The quantum computing helps to perform many computations parallel to each other without interfering each other.

Challenges of Quantum Computing: -

$>$ The main challenge to have quantum computer in an organisation is that it is very costly to implement and not all business can afford it.

$>$ The quantum computers cannot operate accurately in all types of environments as 
they need special environment to operate like cool room temperature and noise free environment.

$>$ Quantum computers are very difficult to implement and design and a lot of challenges is faced by the designers.

Conclusion: - Quantum figuring is the new innovation which further develops basic PC frameworks in to cutting edge frameworks which have the capacity to perform different numerical calculations, material science calculations, data speculations and so on. With conventional PC frameworks it is seen that the capacity of frameworks to perform intense calculations is exceptionally less and subsequently specialists began search to further develop the estimation arrangement of the PC framework so they can be utilized to perform other major troublesome computations which thus will assist the researchers with saving their time and energy which they spend on performing manual logical estimations. Old customary PC frameworks comprehend the language of parallel framework which implies they will initially change over the human language into stream of pieces and bytes and afterward play out the computations. This makes restricted estimations to be performed by the framework. Quantum figuring assists with upgrading the customary PC framework in to propel innovation as it gives the ability to perform high computational power, quick speed which is determined as far as example, utilizes less power and so forth. The quantum registering utilizes the pieces and qubits of quantum hypothesis which makes it conceivable to perform most troublesome estimations. In little conventional PCs, circuits with semiconductors, reasoning doorways, and Integrated Circuits are ridiculous. Thus, quantum figuring uses the subatomic particles like atoms, electrons, photons, and particles as their pieces close by their information of turns and states. They can be superposed and can give more blends. Thus they can run in equivalent using memory really and hence is even more great.

References: -

1.https://www.intechopen.com/online-first/73811

2.https://ictreverse.com/what-are-quantum-computers-andwhy-are-they-important/

3.https://www.visualcapitalist.com/three-types-quantumcomputers/

4.https://research.aimultiple.com/quantum-computinghardware/

5.https://www.e-spincorp.com/the-advantages-anddisadvantage-of-quantum-computing/ 